\title{
The Coal's Layer Temperature Regime under Conditions of Heat Removal of Absorbed Microwave Energy by Radiation and Convection Simultaneously
}

\author{
V. A. Karelin ${ }^{1,2}$, Vl. V. Salomatov ${ }^{1,2}$, Vas. V. Salomatov ${ }^{1}$ \\ ${ }^{1}$ Institute of Thermal Physics, Siberian Branch of the Russian Academy of Sciences, Novosibirsk, Russia \\ ${ }^{2}$ Physics Department, Novosibirsk National Research State University, Novosibirsk, Russia \\ Email:vad2hen@mail.ru, salomatov.vv@mail.ru,vvsalomatov@mail.ru
}

How to cite this paper: Karelin, V.A., Salomatov, Vl.V. and Salomatov, Vas.V. (2018) The Coal's Layer Temperature Regime under Conditions of Heat Removal of Absorbed Microwave Energy by Radiation and Convection Simultaneously. Journal of Computer and Communications, 6, 229-238. https://doi.org/10.4236/jcc.2018.61023

Received: November 19, 2017 Accepted: December 26, 2017 Published: December 29, 2017

\begin{abstract}
Coal is one of the most popular sources of energy. However, it has a relatively low energy efficiency due to high humidity and a greater release of harmful substances during combustion. On the other hand, the coal reserve on earth is estimated at 500 years and the cost is relatively low. This causes the search for new ways of processing coal. One way to efficiently process coal, while reducing the humidity and content of harmful components is microwave treatment. The basic information for microwave exposure to coal is the temperature field. In this paper, an approximate-analytically nonlinear mathematical model for heating a flat coal mass is studied, provided that the absorbed microwave energy is removing by heat radiation and convection simultaneously.
\end{abstract}

\section{Keywords}

Coal, Microwave, Temperature Distribution, Stefan-Boltzmann Law, Convection

\section{Introduction}

At present, there are many experimental confirmations [1] [2] of the prospects of using microwave treatment of coal material. Advantages over conventional processing methods (convective, infrared, conductive, etc.) include the uniformity and volume character of heating; the possibility of concentrating large capacities in certain places; obtaining the required temperature distribution; low inertia; absence of a heat-bearing medium, etc., which significantly increases the 
effectiveness of such an impact on energy coal. These specific features of microwave radiation make it possible to search for optimal heating regimes taking into account changes in the characteristics of coal during the process (humidity, content of other components, temperature, thermophysical and electromagnetic inhomogeneity, etc.) [3]. A comparison was made between the economics of drying by various methods and the microwave advantage was demonstrated [4]. A number of experiments were performed showing the possibility and efficiency of combining different types of drying with microwave drying [5].

Energy costs for evaporation under the influence of microwave radiation are reduced to an average of two times. It is shown that with evaporation of moisture, complete removal of nitrogen from wet coal samples occurs. In addition, there is a reduction of about $50 \%$ sulfur, $50 \%$ mercury, $30 \%$ ash, $50 \%$ chlorine [6].

Even a brief analysis of the application of microwave treatment of coal massifs demonstrates the prospects of its use and development in modern energy technologies, which will significantly improve the environmental safety and efficiency of burning coal fuel.

\section{Problem of Microwave Heating of Plane Coal Massive}

Coal is a dielectric material capable of absorbing electromagnetic radiation from the microwave range and thus forming an internal heat source. In this formulation, it was modeled according to Bouguer's exponential law (Equation (1)). This internal heat source ensures warming up, ignition and burning out of the coal massif. The layer itself has two boundaries. At the top-the mode of heat removal using two mechanisms: radiation and convection (see Figure 1), on the bottom thermal insulation. Radiation is most pronounced at high temperatures $\left(\sim \mathrm{T}^{4}\right)$, convection at lower temperatures $(\sim \mathrm{T})$. However, in the general case, it is necessary to take into account two parallel heat fluxes.

The mathematical model contains the following assumptions:

1) Thermophysical characteristics of coal are constant and isotropic; 2) The initial temperature of the layer is constant along the coordinate; 3) Heat from the surface of the array is removed by heat radiation and convection simultaneously; 4) The non-isothermicity of a coal massif is taken into account.

Taking into account the above assumptions, the system of differential equations of the given problem will be written in the following form:

$$
\begin{gathered}
\frac{\partial T(x, t)}{\partial t}=a \frac{\partial^{2} T(x, t)}{\partial x^{2}}+\frac{q_{v_{0}}}{c \rho} e^{-k x} \\
0 \leq t \leq t_{s}, \quad 0 \leq x \leq l \\
T(x, 0)=T_{0} \\
-\lambda \frac{\partial T(l, t)}{\partial x}=\sigma\left[T^{4}(l, t)-T_{c}^{4}\right]+\alpha\left[T(l, t)-T_{c}\right] \\
\frac{\partial T(0, t)}{\partial x}=0
\end{gathered}
$$




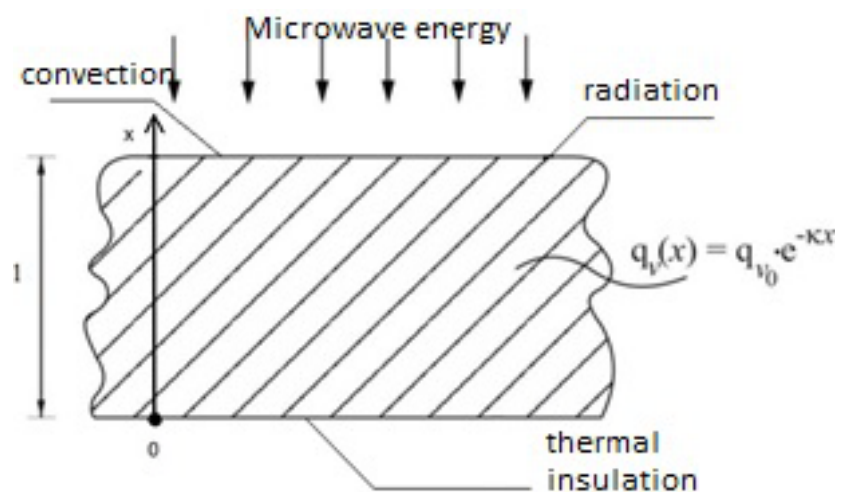

Figure 1. The scheme of the problem of microwave heating with heat dissipation by convection and heat radiation simultaneously.

Here $\mathrm{t}$ is the current time, $\mathrm{s}$; $\mathrm{x}$-current Cartesian coordinate, $\mathrm{m}$; $t_{s}$ - the moment of ignition, $s$; 1 -thickness of the coal massif, $m$; a-coefficient of thermal diffusivity, $\mathrm{m}^{2} / \mathrm{s} ; T(x, t)$ is the current temperature, $K ; \lambda$-coefficient of thermal conductivity, $\mathrm{W} / \mathrm{m} \cdot \mathrm{K} ; \mathrm{c}$-heat capacity, $\mathrm{kJ} / \mathrm{kg} \cdot \mathrm{K} ; \alpha$-Heat transfer coefficient at the upper surface, $\mathrm{W} / \mathrm{m}^{2} \cdot \mathrm{K} ; \rho$-density, $\mathrm{kg} / \mathrm{m}^{3} ; \sigma$-Visible coefficient of thermal radiation, $\mathrm{W} / \mathrm{m}^{2} \cdot \mathrm{K}^{4} ; k$ is the absorption coefficient of microwave radiation, $\mathrm{m}^{-1}$; $\mathrm{T}_{\mathrm{c}}$-ambient temperature, $\mathrm{K}$.

In order to find a generalized solution, we turn to dimensionless variables, choosing as scales the quantities entering into the uniqueness conditions of the problem. To this end, we introduce new variables into problem (1)-(4):

$X=\frac{X}{l}$-dimensionless coordinate,

$F o=\frac{t}{l^{2} / a}-$ Fourier number,

$P o=\frac{q_{v_{0}} l^{2}}{\lambda T_{c}}-$ number of Pomerantsev,

$B u=k l-$ Bouguer's number,

$\theta(X, F o)=\frac{T(x, t)}{T_{c}}-$ dimensionless temperature,

$S k=\frac{\sigma T_{c}^{3} l}{\lambda}-$ Stark number,

$B i=\frac{\alpha l}{\lambda}-$ Bio number.

As a result, we have:

$$
\begin{gathered}
\frac{\partial \theta(X, F o)}{\partial F o}=\frac{\partial^{2} \theta(X, F o)}{\partial X^{2}}+P o \cdot e^{-B u X} \\
\theta(X, 0)=\theta_{0} \\
-\frac{\partial \theta(1, F o)}{\partial X}=S k\left[\theta^{4}(1, F o)-1\right]+B i[\theta(1, F o)-1] \equiv K i(F o) \\
\frac{\partial \theta(0, F o)}{\partial X}=0
\end{gathered}
$$


Problem (5)-(8) does not have a strict analytic solution, since the Stefan-Boltzmann law is nonlinear in (7). However, as shown in our paper [7], it is possible to construct sufficiently effective asymptotics for such problems. Next, we turn to the construction of such asymptotics for small and large values of Fo. To this end, we translate the system (5)-(8) from the region of the originals into the Laplace region of images.

The transformed system looks like this:

$$
\begin{gathered}
s \theta_{L}(X, s)-\theta_{0}=\theta_{L}^{\prime \prime}(X, s)+\frac{P o}{s} e^{-B u X} \\
-\theta_{L}^{\prime}(1, s)=K i_{L}(s) \\
\theta_{L}^{\prime}(0, s)=0
\end{gathered}
$$

We find the solution of this system as the sum of the solution of a homogeneous problem and a solution with allowance for the inhomogeneous term:

$$
\theta_{L}(X, s)=Y_{1}(X, s)+Y_{2}(X, s)
$$

The solution of the homogeneous problem in the general form gives:

$$
Y_{1}(X, s)=A \cdot e^{\sqrt{s} \cdot X}+B \cdot e^{-\sqrt{s} \cdot X}
$$

The nonhomogeneous part must be of the form:

$$
Y_{2}(X, s)=C \cdot e^{-B u \cdot X}+\frac{\theta_{0}}{s}
$$

Next we substitute the general form of the solution

$$
\theta_{L}(X, s)=A \cdot e^{\sqrt{s} \cdot X}+B \cdot e^{-\sqrt{s} \cdot X}+C \cdot e^{-B u \cdot X}+\frac{\theta_{0}}{s}
$$

for an inhomogeneous problem in Equations (9)-(11), we find the constants.

Then the general and particular solution for the Laplace-transformed temperature distribution can be written:

$$
\begin{aligned}
& \theta_{L}(X, s)=\frac{P o \cdot B u}{s^{\frac{3}{2}} \cdot\left(s-B u^{2}\right)}\left[\left(1+\frac{e^{\sqrt{s}}-e^{-B u}}{e^{-\sqrt{s}}-e^{\sqrt{s}}}\right) \cdot e^{\sqrt{s} \cdot X}+\frac{e^{\sqrt{s}}-e^{-B u}}{e^{-\sqrt{s}}-e^{\sqrt{s}}} e^{-\sqrt{s} \cdot X}\right] \\
& -K i_{L}(s) \cdot \Phi(s, X)+\frac{P o}{s\left(s-B u^{2}\right)} e^{-B u X}+\frac{\theta_{0}}{s}
\end{aligned}
$$

Here is Transmission function:

$$
\Phi(s, X)=\frac{\operatorname{ch}(\sqrt{s} X)}{\sqrt{s} \cdot \operatorname{sh} \sqrt{s}}
$$

Next, we turn to the search for asymptotic solutions for small times.

\section{Small Fo (Large $s$ )}

We introduce the notation $Y_{1}(X, s)=-K i(s) \cdot \Phi(s, X)$-the general solution.

$$
\begin{aligned}
& Y_{2}(X, s)=\frac{P o \cdot B u}{\sqrt{s} \cdot s \cdot\left(s-B u^{2}\right)}\left[\left(1+\frac{e^{\sqrt{s}}-e^{-B u}}{e^{-\sqrt{s}}-e^{\sqrt{s}}}\right) \cdot e^{\sqrt{s} \cdot X}+\frac{e^{\sqrt{s}}-e^{-B u}}{e^{-\sqrt{s}}-e^{\sqrt{s}}} e^{-\sqrt{s} \cdot X}\right]+ \\
& +\frac{P o}{s\left(s-B u^{2}\right)} e^{-B u X}+\frac{\theta_{0}}{s}
\end{aligned}
$$


- A particular solution.

Then the system of basic Equations (5)-(8) after the Laplace transform looks as follows:

$$
\begin{gathered}
Y_{1}^{\prime}(X, s)-s Y_{1}(X, s)=0 \\
-Y_{1}^{\prime}(1, s)=K i(s) \\
Y_{1}^{\prime}(0, s)=0
\end{gathered}
$$

We represent the transfer function in the form of an expansion in the large parameter s:

$$
\Phi(s, X)=\frac{\operatorname{ch}(\sqrt{s} X)}{\sqrt{s} \cdot \operatorname{sh} \sqrt{s}} \approx \Psi_{1}(X, s) \frac{e^{-\sqrt{s}}}{\sqrt{s}}+\Psi_{2}(X, s) \frac{e^{-2 \sqrt{s}}}{s}+\ldots
$$

As a result, the general solution in the images becomes:

$$
Y_{1}(X, s)=-K i_{L}(s)\left[\Psi_{1}(X, s) \frac{e^{-\sqrt{s}}}{\sqrt{s}}+\Psi_{2}(X, s) \frac{e^{-2 \sqrt{s}}}{s}+\ldots\right]
$$

Further we substitute the solution $Y_{1}(X, s)$ into the system (9)-(11) and equate the terms with the same degrees of expansion.

As a result, for $\Psi_{1}(X, s)$ we get

$$
\Psi_{1}^{\prime \prime}(X, s)-s \Psi_{1}(X, s)=0
$$

From Equation (10) it follows that

$$
\begin{gathered}
-K i_{L}(s)\left[\Psi_{1}^{\prime}(1, s) \frac{e^{-\sqrt{s}}}{\sqrt{s}}\right]=-K i_{L}(s) \\
\Psi_{1}^{\prime}(1, s)=\frac{\sqrt{s}}{e^{-\sqrt{s}}} \\
\Psi_{1}^{\prime}(0, s)=0
\end{gathered}
$$

The solution of (15) is represented in the form

$$
\Psi_{1}(1, s)=A \cdot \operatorname{sh}(\sqrt{s} X)+B \cdot \operatorname{ch}(\sqrt{s} X)
$$

From the boundary condition (16) it follows that

$$
A=0
$$

As a result, we have:

$$
-K i_{L}(s)\left[\Psi_{1}(X, s) \frac{e^{-\sqrt{s}}}{\sqrt{s}}+\ldots\right]=B \cdot \operatorname{ch}(\sqrt{s} X)
$$

For $X=1$, using (15), we find

$$
B=-\frac{K i(s)}{\sqrt{s} \cdot \operatorname{sh}(\sqrt{s})}
$$

Then

$$
\Psi_{1}(X, s)=-\frac{K i(s) \cdot \operatorname{ch}(\sqrt{s} X)}{\sqrt{s} \cdot \operatorname{sh}(\sqrt{s})}
$$

Further we substitute hyperbolic functions into (19): 


$$
\Psi_{1}(X, s)=-K i(s) \cdot\left(e^{-\sqrt{s}(X+1)}+e^{\sqrt{s}(X-1)}\right)
$$

As a result, the solution in images becomes

$$
\begin{aligned}
& \theta_{L}(X, s)=\frac{P o \cdot B u}{s^{\frac{3}{2}} \cdot\left(s-B u^{2}\right)}\left[\left(1+\frac{e^{\sqrt{s}}-e^{-B u}}{e^{-\sqrt{s}}-e^{\sqrt{s}}}\right) \cdot e^{\sqrt{s} \cdot X}+\frac{e^{\sqrt{s}}-e^{-B u}}{e^{-\sqrt{s}}-e^{\sqrt{s}}} e^{-\sqrt{s} \cdot X}\right] \\
& -\frac{K i_{L}(s)}{\sqrt{s}} \cdot\left(e^{-\sqrt{s}(X+2)}+e^{\sqrt{s}(X-2)}\right)+\frac{P o}{s\left(s-B u^{2}\right)} e^{-B u X}+\frac{\theta_{0}}{s}
\end{aligned}
$$

Then we carry out the inverse Laplace transform. For large $s$ it is permissible to assume that $s \gg B u$ and

$$
L^{-1}\left[\frac{P o}{s^{2}} \cdot e^{-B u X}\right]=P o e^{-B u X} F O
$$

For large s, the expression

$$
\frac{P o \cdot B u}{\sqrt{s} \cdot s \cdot\left(s-B u^{2}\right)}\left[\left(1+\frac{e^{\sqrt{s}}-e^{-B u}}{e^{-\sqrt{s}}-e^{\sqrt{s}}}\right) \cdot e^{\sqrt{s} \cdot X}+\frac{e^{\sqrt{s}}-e^{-B u}}{e^{-\sqrt{s}}-e^{\sqrt{s}}} e^{-\sqrt{s} \cdot X}\right]
$$

goes into

$$
-\frac{P o \cdot B u}{\sqrt{s} \cdot s^{2}} e^{-\sqrt{s} \cdot X}
$$

Let

$$
N(X, F O)=L^{-1}\left[-\frac{P o \cdot B u}{\sqrt{s} \cdot s^{2}} e^{-\sqrt{s} \cdot X}\right]
$$

Then the final solution is written in convolution

$$
\begin{aligned}
& \theta(X, F O)=-\int_{0}^{F o} \frac{K i(\eta)}{\sqrt{\pi(F O-\eta)}} \cdot\left(e^{-\frac{(X+2)^{2}}{4(F o-\eta)}}+e^{\frac{(X-2)^{2}}{4(F o-\eta)}}\right) d \eta \\
& +P o \cdot e^{-B u X} F_{O}+N(X, F O)
\end{aligned}
$$

To obtain an explicit calculation expression, we expand $K i(\eta)$ in the Taylor series near $\eta \approx F o: K i(\eta)=K i(F o)+(\eta-F o) \cdot K i(F o)+\ldots$

Limited to the first term of the expansion, we have

$$
\begin{aligned}
& \theta(X, F O)=-K i(F O) \int_{0}^{F o} \frac{1}{\sqrt{\pi(F O-\eta)}} \cdot\left(e^{-\frac{(X+2)^{2}}{4(F o-\eta)}}+e^{\frac{(X-2)^{2}}{4(F o-\eta)}}\right) d \eta \\
& +P O \cdot e^{-B u X} F_{O}+N(X, F O)
\end{aligned}
$$

After taking the integral, we get:

$$
\begin{aligned}
& \theta(X, F o)=\left(S k\left(\theta^{4}(1, F o)-1\right)+B i(\theta(1, F o)-1)\right) \\
& \cdot\left(\frac{X}{\sqrt{\pi}}-\frac{X+2}{\sqrt{\pi} \cdot \frac{X+2}{\sqrt{F O}}} \cdot\left[\frac{\sqrt{\pi}(X+2)}{2 \sqrt{F O}} \operatorname{erf}\left(\frac{X+2}{2 \sqrt{F O}}\right)+e^{-\left(\frac{(X+2)}{2 \sqrt{F o}}\right)^{2}}\right]\right.
\end{aligned}
$$

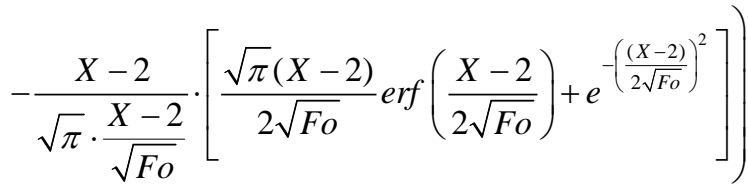

$$
\begin{aligned}
& +P o \cdot e^{-B u X} F o+\theta_{0}+N(X, F o)
\end{aligned}
$$


The boundary temperature is found by substituting $X=1$ in (30):

$$
\begin{aligned}
& \theta(1, F o)=\left(S k\left(\theta^{4}(1, F o)-1\right)+B i(\theta(1, F o)-1)\right) \\
& \cdot\left(\frac{1}{\sqrt{\pi}}-\frac{3}{2} \operatorname{erf}\left(\frac{3}{2 \sqrt{F O}}\right)+\frac{1}{2} \operatorname{erf}\left(-\frac{1}{2 \sqrt{F O}}\right)-\frac{\sqrt{F O}}{\sqrt{\pi}}\left[e^{-\left(\frac{3}{2 \sqrt{F o}}\right)^{2}}+e^{\left.\left.-\left(\frac{1}{2 \sqrt{F o}}\right)^{2}\right]\right)}\right.\right. \\
& +P O \cdot e^{-B u} F O+\theta_{0}+N(1, F o)
\end{aligned}
$$

As a result, the surface temperature is calculated by the following expression:

$$
\theta(1, F O)=\frac{\sqrt{b+c} \pm \sqrt{-b-c+2(1-B i)(S k \sqrt{b+c})^{-1}}}{2}+a
$$

Here are new variables:

$$
\begin{gathered}
a=+P o \cdot e^{-B u} F O+\theta_{0}+N(1, F o) \\
b=\frac{4 \cdot 2^{\frac{1}{3}} a}{\left(27 \cdot(B i \cdot d-1)^{2} S k \cdot d+\sqrt{729(B i \cdot d-1)^{4}(S k \cdot d)^{2}-6912 \cdot a^{3}(S k \cdot d)^{3}}\right)^{\frac{1}{3}}} \\
c=\frac{\left(27 \cdot(B i \cdot d-1)^{2} S k \cdot d+\sqrt{729(B i \cdot d-1)^{4}(S k \cdot d)^{2}-6912 \cdot a^{3}(S k \cdot d)^{3}}\right)^{\frac{1}{3}}}{3 \cdot 2^{\frac{1}{3}} S k \cdot d} \\
d=\left(\frac{1}{\sqrt{\pi}}-\frac{3}{2} \operatorname{erf}\left(\frac{3}{2 \sqrt{F O}}\right)+\frac{1}{2} \operatorname{erf}\left(-\frac{1}{2 \sqrt{F O}}\right)-\frac{\sqrt{F o}}{\sqrt{\pi}}\left[e^{-\left(\frac{3}{2 \sqrt{F o}}\right)^{2}}+e^{\left.-\left(\frac{1}{2 \sqrt{F o}}\right)^{2}\right]}\right]\right)
\end{gathered}
$$

\section{Large Fo (Small $s$ )}

We take the inverse transformation from the term with the source as we do on previous stage, but now we calculating expressions with assumption of small $\mathrm{s}$. Let's set new variable:

$$
M(X, F o)=L^{-1}\left[\frac{P o \cdot B u}{s^{2} B u^{2}}\left(1-e^{-B u}\right)\right]=\frac{\left(1-e^{-B u}\right) F o \cdot P o}{B u}
$$

We shall construct the asymptotics for large times-for this purpose we expand the transfer function in a series with respect to the small parameter s:

$$
\Phi(s, X)=\varphi_{0}(s, X)+s \varphi_{1}(s, X)+s^{2} \varphi_{2}(s, X)+\cdots
$$

Substituting (27) into the basic Equation (9) and equating the terms with the same powers of $\mathrm{s}$, we obtain a system for determining $\varphi_{0}, \varphi_{1}, \varphi_{2}$

The system that is used for this:

$$
\begin{gathered}
Y_{1}^{\prime \prime}(X, s)-s \cdot Y_{1}(X, s)=0 \\
-Y_{1}^{\prime}(1, s)=K i_{L}(s) \\
Y_{1}^{\prime}(0, s)=0
\end{gathered}
$$

As a result, we get 


$$
\varphi_{0}^{\prime \prime}(X, s)=0, \varphi_{1}^{\prime \prime}(X, s)=\varphi_{0}, \varphi_{2}^{\prime \prime}(X, s)=\varphi_{1}
$$

Each equation in (28) requires 2 boundary conditions for finding two constants.

The boundary condition:

$$
\left.\varphi_{0}^{\prime}\right|_{X=0}=0,\left.\varphi_{1}^{\prime}\right|_{X=0}=0,\left.\varphi_{2}^{\prime}\right|_{X=0}=0
$$

The second constant is found from the integral relations:

$$
\int_{0}^{1} \varphi_{0}^{\prime \prime} d X=0, \int_{0}^{1} \varphi_{1}^{\prime \prime} d X=\int_{0}^{1} \varphi_{0} d X, \int_{0}^{1} \varphi_{2}^{\prime \prime} d X=\int_{0}^{1} \varphi_{1} d X
$$

We solve the system, finding for the asymptotics in the originals with allowance for the two terms of the series (27). As a result, we have

$$
\begin{aligned}
& \theta_{L}(X, F o) \approx-\int_{0}^{F o} K i(\eta) d \eta+K i(F o) \frac{1-3 X^{2}}{6} \\
& +P o \cdot F o \cdot e^{-B u X}+\theta_{0}+M(X, F o)
\end{aligned}
$$

The unknown surface temperature $\theta(1, F o)$ is found from the Voltaire integral equation of the second kind, while denoting $\theta_{w}(F o)=\theta(1, F o)$ :

$$
\begin{aligned}
& \theta_{w}(F o) \approx-\int_{0}^{F o}\left[\operatorname{Bi}\left(\theta_{w}(\eta)-1\right)+\operatorname{Sk}\left(\theta_{w}{ }^{4}(\eta)-1\right)\right] d \eta \\
& -\frac{1}{3}\left[\operatorname{Bi}\left(\theta_{w}(F o)-1\right)+S k\left(\theta_{w}{ }^{4}(F o)-1\right)\right]+P o \cdot F o \cdot e^{-B u}+\theta_{0}+M(1, F o)
\end{aligned}
$$

The solution of (32) is

$$
3 S k \cdot F o=F_{1}\left[\theta_{w}^{*}, \frac{B i}{S k}\right]-F_{1}\left[\theta_{w}, \frac{B i}{S k}\right]
$$

where the function $F_{1}$ has the form

$$
\begin{aligned}
& F_{1}\left[\theta_{w}\right]=-\frac{A}{4 \sqrt{2} \cdot B^{\frac{3}{4}}}\left(2 \cdot \arctan \left[1-\frac{\sqrt{2} \cdot \theta_{w}}{B^{1 / 4}}\right]-2 \cdot \arctan \left[1+\frac{\sqrt{2} \cdot \theta_{w}}{B^{1 / 4}}\right]\right. \\
& \left.+\ln \left(\frac{\theta_{w}{ }^{2}-\sqrt{2} \cdot \theta_{w} \cdot B^{1 / 4}+\sqrt{B}}{\theta_{w}{ }^{2}+\sqrt{2} \cdot \theta_{w} \cdot B^{1 / 4}+\sqrt{B}}\right)\right)+S k \cdot \ln \left(\theta_{w}{ }^{4}+B\right)
\end{aligned}
$$

Here $\theta_{w}^{*}$ is the initial value in the integral Equation (32). We find it substituting $\mathrm{Fo}=0$ :

$$
\theta_{w}^{*}=-\frac{1}{3}\left[\operatorname{Bi}\left(\theta_{w}^{*}-1\right)+\operatorname{Sk}\left(\theta_{w}^{* 4}-1\right)\right]+\theta_{0}
$$

The solution of (34) is:

$$
\theta_{w}^{*}=\frac{1}{2} \sqrt{-A+B}-\frac{1}{2} \sqrt{A-B+C}
$$

where

$$
A=\frac{4\left(\frac{2}{3}\right)^{\frac{1}{3}}\left(B i+S k+3 \theta_{0}\right)}{\left(9(3+B i)^{2} \cdot S k+\sqrt{3\left(27(3+B i)^{4} \cdot S k^{2}+256 \cdot S k^{3}\left(B i+S k+3 \theta_{0}\right)^{3}\right)}\right)^{\frac{1}{3}}}
$$




$$
\begin{gathered}
B=\frac{\left(9(3+B i)^{2} \cdot S k+\sqrt{3\left(27(3+B i)^{4} \cdot S k^{2}+256 \cdot S k^{3}\left(B i+S k+3 \theta_{0}\right)^{3}\right)}\right)^{\frac{1}{3}}}{2^{\frac{1}{3}} \cdot 3^{\frac{2}{3}} \cdot S k} \\
C=\frac{2 \cdot(3+B i)}{S k \cdot \sqrt{-A+B}}
\end{gathered}
$$

Knowing the surface temperature, you can calculate the required temperature:

$$
\begin{aligned}
& \vartheta_{L}(X, F o) \approx \theta_{w}(F o)-\left(B i \cdot\left(\theta_{w}(F o)-1\right)+S k\left(\theta_{w}{ }^{4}(F o)-1\right)\right) \cdot \frac{1-3 X}{6} \ldots \\
& +P o \cdot F o \cdot e^{-B u X}
\end{aligned}
$$

A typical temperature distribution obtained from this solution is demonstrated on Figure 2.

The stationary solution for large times is found from the basic Equation (5) and looks as follows:

$$
\theta(X, \infty)=\frac{P o}{B u^{2}} e^{-B u X}
$$

\section{Conclusions}

This article is a construction of a nonlinear model of heating a coal layer by microwave energy. At the same time internal heat sources are generated inside, which are modeled according to Bouguer's law. On the upper surface, a heat release is prescribed according to the law of heat radiation and convection. Since they are decisive in the process of heating the coal to the ignition temperature. The lower boundary is considered to be insulated in the problem. The task itself to simplify the recording of the solution has been translated into dimensionless variables. The problem was analyzed using the Laplace transform. Because of the Stefan-Boltzmann law, the problem is nonlinear and, generally speaking, does

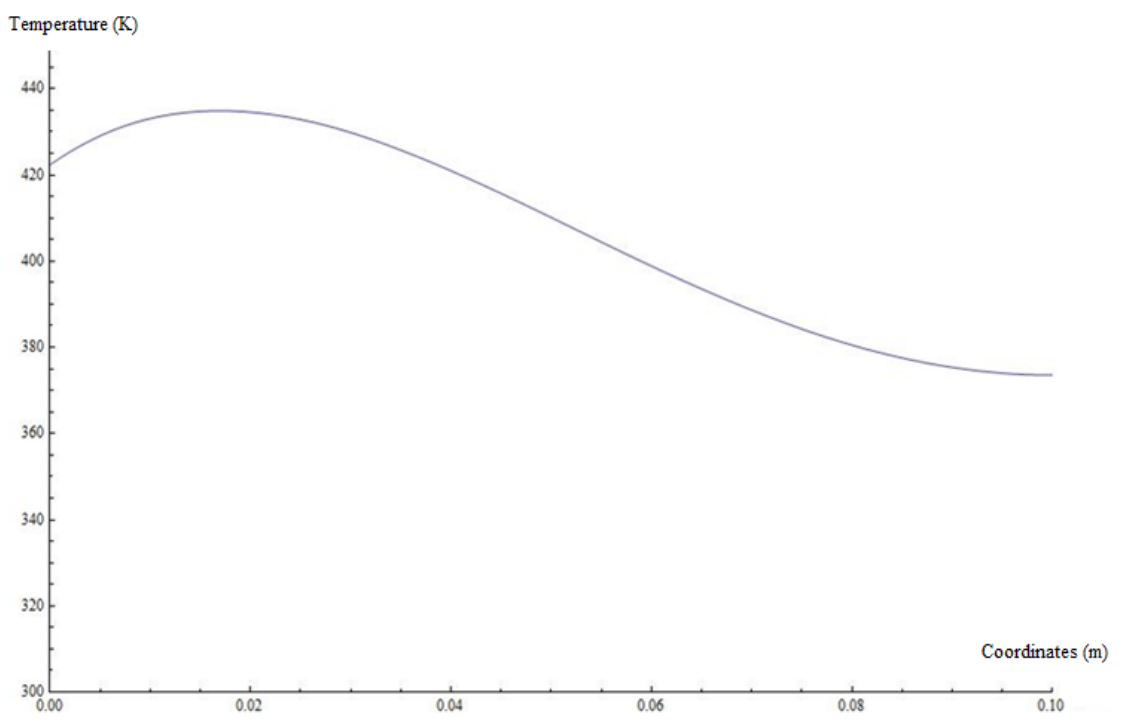

Figure 2. Temperature distribution over the depth of the layer. 
not have a rigorous analytical solution. To do this, we searched for asymptotic solutions for large and small times.

The resulting temperature fields will be used in future to calculate thermal stresses, find the moment of ignition, optimum conditions, conduct microwave processing, taking into account ecology, energy saving, safety, etc.

\section{Funding}

The reported study was funded by Russian Foundation for Basic Research (RFBR) according to the research project No. 17-08-00752.

\section{References}

[1] Salomatov, Vl.V., Sladkov, S.O., Pashchenko, S.E. and Salomatov, Vas.V. (2013) Phenomenological Models of Microwave Heating of a Flat Coal Mass with Release of Absorbed Heat by the Convection Law. Journal of Engineering Thermophysics, 22, 134-142. https://doi.org/10.1134/S1810232813020057

[2] Salomatov, V.V., Sladkov, S.O. and Pashchenko, S.E. (2012) Microwave Technologies in Coal Power Engineering. IFH, 85. 535-549.

[3] Pokras, S.M., Kolesnichenko, Yu.F., Kuryshev, V.A. and Vasiliev, K.B. (1991) On the Possibility of Using Microwave Energy to Solve Problems Arising from the Combustion of Solid Fuels. Proceedings of MRTI, Radiophysics Series, 266-273.

[4] Salomatov, Vl.V., Sladkov, S.O., Paschenko, S.E. and Salomatov, You.V. (2012) Application of Microwave in Coal Heat Power Engineering. Collection of Scientific Works of III Vseros. Scientific and Practical Conference with International Participation "Thermophysical Fundamentals of Energy Technologies", Publishing House of Tomsk University, Tomsk, 8-23.

[5] Khaydurova, A.A. (2010) Microwave Drying of Brown Coals and Increase of Their Technological Characteristics. Izv. Universities. Problems of Energy. Publishing House KGEU, Kazan, 31-35.

[6] Patent USA (2007) 0295.590 Methods and Systems for Improving the Properties of Solid Fuels.

[7] Salomatov, Vl.V., Karelin, V.A. and Salomatov, Vas.V. (2016) Mathematical Models of Microwave Heating of a Coal Mass with Release of Absorbed Energy. Journal of Engineering Thermophysics, 25. 